\title{
Linear Image Reconstruction by Sobolev Norms on the Bounded Domain
}

\author{
Bart J. Janssen · Remco Duits
}

Received: 14 September 2007 / Accepted: 2 July 2008 / Published online: 19 August 2008

(C) The Author(s) 2008. This article is published with open access at Springerlink.com

\begin{abstract}
The reconstruction problem is usually formulated as a variational problem in which one searches for that image that minimizes a so called prior (image model) while insisting on certain image features to be preserved. When the prior can be described by a norm induced by some inner product on a Hilbert space, the exact solution to the variational problem can be found by orthogonal projection. In previous work we considered the image as compactly supported in $\mathbb{L}_{2}\left(\mathbb{R}^{2}\right)$ and we used Sobolev norms on the unbounded domain including a smoothing parameter $\gamma>0$ to tune the smoothness of the reconstructed image. Due to the assumption of compact support of the original image, components of the reconstructed image near the image boundary are too much penalized. Therefore, in this work we minimize Sobolev norms only on the actual image domain, yielding much better reconstructions (especially for $\gamma \gg 0$ ). As an example we apply our method to the reconstruction of singular points that are present in the scale space representation of an image.
\end{abstract}

Keywords Reconstruction - Image reconstruction - Scale space $\cdot$ Deep structure $\cdot$ Generalized sampling $\cdot$ Bounded domain $\cdot$ Sobolev space $\cdot$ Interpolation $\cdot$ Singular point

\section{B.J. Janssen $(\varangle) \cdot$ R. Duits}

Department of Mathematics and Department of Biomedical Engineering, Eindhoven University of Technology, Eindhoven,

The Netherlands

e-mail: b.j.janssen@tue.nl

R. Duits

e-mail: r.duits@tue.nl

\section{Introduction}

One of the fundamental problems in signal processing is the reconstruction of a signal from its samples. In 1949 Shannon published his work on signal reconstruction from its equispaced ideal samples (Shannon 1949). Many generalizations (Papoulis 1977; Unser 2000) and applications (Kybic et al. 2002; Candes et al. 2006) followed thereafter.

Reconstruction from differential structure of scale space interest points, first introduced by Nielsen and Lillholm (2001), is an interesting instance of the reconstruction problem, since the samples are non-uniformly distributed over the image they were obtained from and the filter responses of the filters do not necessarily coincide. Several linear and non-linear methods (Nielsen and Lillholm 2001; Janssen et al. 2006; Kybic et al. 2002; Lillholm et al. 2003) appeared in literature which all search for an image that (1) is indistinguishable from its original when observed through the filters the features were extracted with, and (2) simultaneously minimizes a certain prior. If such a prior is a norm of Sobolev type on the unbounded domain one can obtain visually attractive reconstructions while retaining linearity, as we have shown in earlier work (Janssen et al. 2006; Duits 2005). However, boundaries often cause problems to signal processing algorithms (see e.g. Mallat 1998, Chap. 7.5 or Daubechies 1992, Chap. 10.7) and should be handled with care.

The problem that appears in the unbounded domain reconstruction method is illustrated in Fig. 1. In this figure the left image is a reconstruction from differential structure obtained from a concatenation of (mirrored) versions of Lena's eye. The input image is depicted on the right of Fig. 1 and is still considered as a compactly supported element in $\mathbb{L}_{2}\left(\mathbb{R}^{2}\right)$, as required in our previous work (Janssen et al. 2006). So the mirroring has nothing to do with 


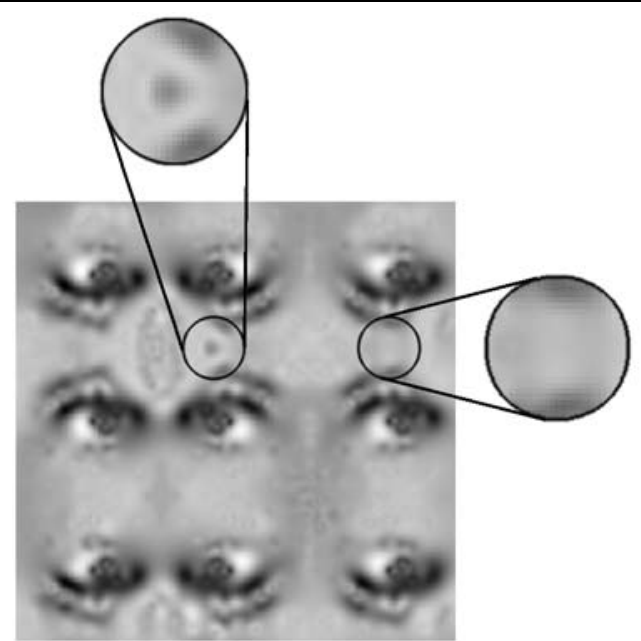

Fig. 1 The left image shows a reconstruction from differential structure obtained from the right image using the unbounded domain reconstruction method as presented in our previous work (Janssen et al. 2006). The upper circle in the right image shows a detail of an area near the center of the image and the circle on the right shows a corresponding area near the boundary of the image. One would expect

boundary conditions. In this particular case we observe that our previous work shows limitations. If the reconstruction method on the unbounded domain would work properly, details that appear in the center of the image are also expected to appear at the corresponding locations near the boundary of the image. This is, however, not the case. To clarify this observation we depicted two magnifications of corresponding positions in the image. The upper circle in Fig. 1 contains a magnification of a part taken from the center of the reconstructed image. This will henceforth be called "magnification $a$ ". The circle on the right contains a magnification of a corresponding patch near the boundary and is referred to as "magnification $b$ ". Note that magnification $a$ contains details that magnification $b$ does not contain.

This problem can be attributed to the fact that kernels, associated to the image-features, partly lay outside the image domain and are "penalized" by the energy minimization methods on the unbounded domain. As a result the reconstructed image shows defects, in particular near the boundary. Even kernels that are attached to features close to the center of the image are unnecessarily suppressed by the energy minimization formulated on the unbounded domain. So the reconstruction depicted in magnification $a$ still suffers from a boundary problem, although less visible than the reconstruction of magnification $b$.

A first rough approach to tackle this problem could be to extend the image symmetrically in all directions and cut out the center after reconstruction. This results in an increase in computation time and it still does not solve the problem in a rigorous manner. Instead, in this article we solve this problem by considering bounded domain Sobolev norms. An ad-

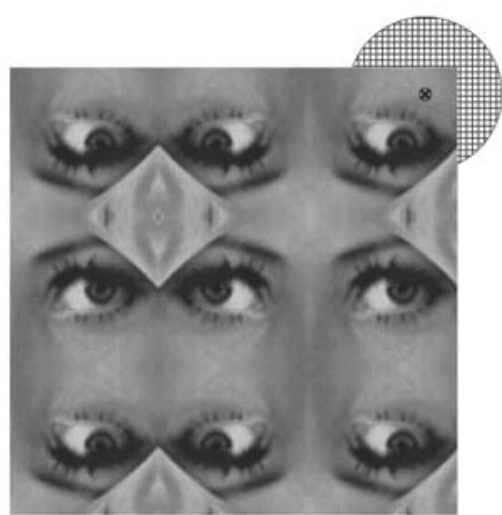

that the same details would appear in both circles. However, this is not the case since kernels that are associated to image-features partly lay outside the image domain and are, as a consequence, penalized by the energy minimization methods that are defined on the unbounded domain. This is illustrated by a marked circle, placed on top of the input image that is displayed on the right

ditional advantage of our method is that we can enforce a much higher degree of regularity than the unbounded domain counter part. Furthermore, we give an interpretation of the 2 parameters that appear in the reconstruction framework in terms of filtering by a low-pass Butterworth filter. This allows for a good intuition on how to choose these parameters.

\section{Theory}

In order to avoid the problem discussed in the introduction and illustrated in Fig. 1 we restrict the reconstruction problem to the domain $\Omega \subset \mathbb{R}^{2}$ that is defined as the support of the image $f \in \mathbb{L}_{2}\left(\mathbb{R}^{2}\right)$ from which the features $\left\{c_{p}(f)\right\}_{1}^{P}, c_{p}(f) \in \mathbb{R}$ are extracted. Recall that the $\mathbb{L}_{2}(\Omega)$ inner product on the domain $\Omega \subset \mathbb{R}^{2}$ for $f, g \in \mathbb{L}_{2}(\Omega)$ is given by

$(f, g)_{\mathbb{L}_{2}(\Omega)}=\int_{\Omega} \overline{f(x)} g(x) \mathrm{d} x$.

A feature $c_{p}(f)$ is obtained by taking the inner product of the $p$ th filter $\psi_{p} \in \mathbb{L}_{2}(\Omega)$ with the image $f \in \mathbb{L}_{2}(\Omega)$,

$c_{p}(f)=\left(\psi_{p}, f\right)_{\mathbb{L}_{2}(\Omega)}$.

An image $g \in \mathbb{L}_{2}(\Omega)$ is equivalent to the image $f$ if they share the same features, $\left\{c_{p}(f)\right\}_{p=1}^{P}=\left\{c_{p}(g)\right\}_{p=1}^{P}$, which is expressed in the following equivalence relation for $f, g \in \mathbb{L}_{2}(\Omega)$ :

$f \sim g \quad \Leftrightarrow \quad\left(\psi_{p}, f\right)_{\mathbb{L}_{2}(\Omega)}=\left(\psi_{p}, g\right)_{\mathbb{L}_{2}(\Omega)}$

for all $p=1, \ldots, P$. 
Next, we introduce the Sobolev space of order $2 k$ on the domain $\Omega$,

$\mathbb{H}^{2 k}(\Omega)=\left\{\left.f \in \mathbb{L}_{2}(\Omega)|| \Delta\right|^{k} f \in \mathbb{L}_{2}(\Omega)\right\}, \quad k>0$.

The completion of the space of $2 k$-differentiable functions on the domain $\Omega$ that vanish on the boundary of its domain $\partial \Omega$ is given by

$\mathbb{H}_{0}^{2 k}(\Omega)=\left\{f \in \mathbb{H}^{2 k}(\Omega)|f|_{\partial \Omega}=0\right\}, \quad k>\frac{1}{2}$.

Now $\mathbb{H}_{0}^{2 k, \gamma}(\Omega)$ denotes the normed space obtained by endowing $\mathbb{H}_{0}^{2 k}(\Omega)$ with the following inner product,

$$
\begin{aligned}
(f, g)_{\mathbb{H}_{0}^{2 k, \gamma}(\Omega)} & =(f, g)_{\mathbb{L}_{2}(\Omega)}+\gamma^{2 k}\left(|\Delta|^{\frac{k}{2}} f,|\Delta|^{\frac{k}{2}} g\right)_{\mathbb{L}_{2}(\Omega)} \\
& =(f, g)_{\mathbb{L}_{2}(\Omega)}+\gamma^{2 k}\left(|\Delta|^{k} f, g\right)_{\mathbb{L}_{2}(\Omega)},
\end{aligned}
$$

for all $f, g \in \mathbb{H}_{0}^{2 k}(\Omega)$ and $\gamma \in \mathbb{R}^{+}$.

The solution to the reconstruction problem is the image $g$ of minimal $\mathbb{H}_{0}^{2 k, \gamma}$-norm that shares the same features with the image $f \in \mathbb{H}_{0}^{2 k, \gamma}(\Omega)$ from which the features $\left\{c_{p}(f)\right\}_{p=1}^{P}$ were extracted. The reconstructed image $g$ is found by an orthogonal projection, within the space $\mathbb{H}_{0}^{2 k, \gamma}(\Omega)$, of $f$ onto the subspace $V$ spanned by the filters $\kappa_{p}$ that correspond to the $\psi_{p}$ filters,

$\arg \underset{g \sim f}{\min }\|g\|_{\mathbb{H}_{0}^{2 k, \gamma}(\Omega)}^{2}=\mathbb{P}_{V} f$,

as shown in previous work (Janssen et al. 2006). The filters $\kappa_{p} \in \mathbb{H}_{0}^{2 k, \gamma}(\Omega)$ are given by

$\kappa_{p}=\left(I+\gamma^{2 k}|\Delta|^{k}\right)^{-1} \psi_{p}$.

As a consequence $\left(\kappa_{p}, f\right)_{\mathbb{H}_{0}^{\left.2 k, \gamma_{(}\right)}}=\left(\psi_{p}, f\right)_{\mathbb{L}_{2}(\Omega)}$ for all $f$ for $(p=1 \ldots P)$. Here we assumed that $f \in \mathbb{H}^{2 k}(\Omega)$. However, it suffices to take $f \in \mathbb{L}_{2}(\Omega)$ if $\psi$ satisfies certain regularity conditions. The interested reader can find the precise conditions and further details in Duits (2005).

The two parameters $\gamma$ and $k$ that appear in the reconstruction problem, allow for an interesting interpretation. If $\Omega=\mathbb{R}$, the fractional operator $\left(I+\gamma^{2 k}|\Delta|^{k}\right)^{-1}$ can be written as

$$
\begin{aligned}
(I & \left.+\gamma^{2 k}|\Delta|^{k}\right)^{-1} f \\
& =\mathcal{F}^{-1}\left(\omega \mapsto\left(1+\gamma^{2 k}|\omega|^{2 k}\right)^{-1}(\mathcal{F} f)(\omega)\right)
\end{aligned}
$$

for all $f \in \mathbb{H}^{2 k}$ and $\omega \in \mathbb{R}$. Here $\mathcal{F}: \mathbb{L}_{2}(\mathbb{R}) \rightarrow \mathbb{L}_{2}(\mathbb{R})$ denotes Fourier transformation, which is defined almost everywhere as

$(\mathcal{F} f)(\omega)=\frac{1}{\sqrt{2 \pi}} \int_{-\infty}^{\infty} f(x) e^{-i \omega x} \mathrm{~d} x$.

Therefore it is equivalent to filtering with the classical lowpass Butterworth filter (Butterworth 1930) of order $2 k$ and cut-off frequency $\omega_{0}=\frac{1}{\gamma}$. The Fourier transform of this filter is defined as

$B_{2 k}\left(\frac{\omega}{\omega_{0}}\right)=\frac{1}{1+\left|\frac{\omega}{\omega_{0}}\right|^{2 k}}$.

The filter response of the Butterworth filter is shown in Fig. 2. One can observe the order of the filter controls how well the ideal low-pass filter is approximated and the effect of $\gamma$ on the cut-off frequency.

\subsection{Spectral Decomposition}

In this section we set $k=1$ and investigate the Laplace operator on the bounded domain: $\Delta: \mathbb{H}_{0}^{2}(\Omega) \rightarrow \mathbb{L}_{2}(\Omega)$. This is a bounded operator, since $\|\Delta f\|_{\mathbb{L}_{2}(\Omega)} \leq 1\|f\|_{\mathbb{H}_{0}^{2}(\Omega)}$ for all $f \in \mathbb{H}_{0}^{2}(\Omega)$, and its right inverse is given by the minus Dirichlet operator:

Definition 1 (Dirichlet Operator) The Dirichlet operator $\mathcal{D}$ is given by

$g=\mathcal{D} f \Leftrightarrow\left\{\begin{array}{l}\Delta g=-f \\ \left.g\right|_{\partial \Omega}=0\end{array}\right.$

with $f \in \mathbb{L}_{2}(\Omega)$ and $g \in \mathbb{H}_{0}^{2}(\Omega)$.

The Green's function $G: \Omega \times \Omega \rightarrow \mathbb{R}$ of the Dirichlet operator is given by

$\left\{\begin{array}{l}\Delta G(\mathbf{x}, \cdot)=-\delta_{\mathbf{x}} \\ \left.G(\mathbf{x}, \cdot)\right|_{\partial \Omega}=0\end{array}\right.$

for fixed $\mathbf{x} \in \Omega$. On the domain $[-a, a] \times[0, b]$ the closed form solution is given by (see the Appendix)

$G_{a, b}(\mathbf{x}, \mathbf{y})=-\frac{1}{2 \pi} \log \left|\frac{\operatorname{sn}\left(x_{1} \frac{z(1, \tilde{k})}{a}+i x_{2} \frac{z\left(1, \sqrt{1-\tilde{k}^{2}}\right)}{b}, \tilde{k}\right)-\operatorname{sn}\left(y_{1} \frac{z(1, \tilde{k})}{a}+i y_{2} \frac{z\left(1, \sqrt{1-\tilde{k}^{2}}\right)}{b}, \tilde{k}\right)}{\operatorname{sn}\left(x_{1} \frac{z(1, \tilde{k})}{a}+i x_{2} \frac{z\left(1, \sqrt{1-\tilde{k}^{2}}\right)}{b}, \tilde{k}\right)-\operatorname{sn}\left(y_{1} \frac{z(1, \tilde{k})}{a}+i y_{2} \frac{z\left(1, \sqrt{1-\tilde{k}^{2}}\right)}{b}, \tilde{k}\right)}\right|$. 
Fig. 2 The filter response of a Butterworth filter, cf. (11). On the left, $\gamma$ is kept constant and the filter responses for different $k>0$ are shown. On the right, the order of the filter, $2 k$, is kept constant and the filter responses for different $\gamma>0$ are shown

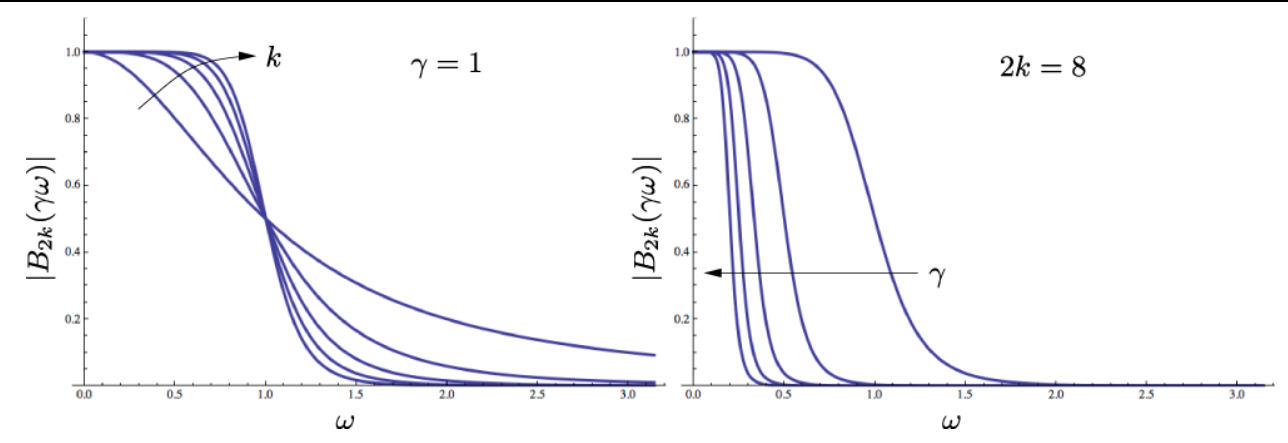

Here $\mathbf{x}=\left(x_{1}, x_{2}\right), \mathbf{y}=\left(y_{1}, y_{2}\right) \in \Omega, \tilde{k} \in \mathbb{R}$ is determined by the aspect ratio of the rectangular domain $\Omega$, sn denotes the Jacobi-elliptic function (Gradshteyn and Ryzhik 1994), (Whittaker and Watson 1946, Chap. XXII), and

$z(1, \tilde{k})=\int_{0}^{1} \frac{\mathrm{d} t}{\sqrt{1-t^{2}} \sqrt{1-\tilde{k}^{2} t^{2}}}$.

In the Appendix we derive (14), and show how to obtain $\tilde{k}$. Figure 3 shows a graphical representation of this non-isotropic Green's function for a square domain $(\tilde{k} \approx$ $0.1716)$. Notice this function vanishes at its boundaries and is, in the center of the domain, very similar to the isotropic fundamental solution on the unbounded domain (Duchon 1977). In the Appendix we put a relation between the fundamental solution of the Laplace operator on the unbounded domain and the Green's function on the bounded domain with Dirichlet boundary conditions. In terms of regularization this means the Dirichlet operator smooths inwards the image but never "spills" over the border of the domain $\Omega$.

When the Dirichlet operator, as defined in Definition 1, is expressed by means of its Green's function, which is presented in (14),

$$
\begin{gathered}
(\mathcal{D} f)(\mathbf{x})=\int_{\Omega} G(\mathbf{x}, \mathbf{y}) f(\mathbf{y}) \mathrm{d} \mathbf{y}, \\
f \in \mathbb{L}_{2}(\Omega), \mathcal{D} f \in \mathbb{H}_{0}^{2}(\Omega)
\end{gathered}
$$

one can verify it extends to a compact, self-adjoint operator on $\mathbb{L}_{2}(\Omega)$. As a consequence, by the spectral decomposition theorem of compact self-adjoint operators (Yosida 1980), we can express the Dirichlet operator in an orthonormal basis of eigenfunctions. The normalized eigenfunctions $f_{m n}$ with corresponding eigenvalues $\lambda_{m n}$ of the Laplace operator $\Delta: \mathbb{H}_{0}^{2}(\Omega) \rightarrow \mathbb{L}_{2}(\Omega)$ are given by

$$
\begin{aligned}
& f_{m n}(x, y)=\sqrt{\frac{1}{a b}} \sin \left(\frac{n \pi x}{a}\right) \sin \left(\frac{m \pi y}{b}\right), \\
& \lambda_{m n}=-\left(\left(\frac{n \pi}{a}\right)^{2}+\left(\frac{m \pi}{b}\right)^{2}\right),
\end{aligned}
$$

$(x, y) \in \Omega$ with $\Omega=[0, a] \times[0, b]$ and $m, n \in \mathbb{N}$. These functions can be found by the method of separation
(Kreyszig 1993). Since $\Delta \mathcal{D}=-I$, the eigenfunctions of the Dirichlet operator coincide with those of the Laplace operator (17), and its corresponding eigenvalues are the inverse of the eigenvalues of the Laplace operator (18).

\subsection{Scale Space on the Bounded Domain}

The spectral decomposition presented in the previous subsection, by (17) and (18), will now be applied to the construction of a scale space on the bounded domain. We will follow the second author's previous work (Duits et al. 2003) on scale spaces on the bounded domain. Here we recall from (Duits et al. 2003) that Neumann boundary conditions are required in order to maintain most scale space axioms (Duits et al. 2004). However, here we shall first consider Dirichlet boundary conditions. In Sect. 2.4 we will also consider Neumann boundary conditions. Before we show how to obtain a Gaussian scale space representation of an image on the bounded domain we find, as suggested by Koenderink (1984), the image $h \in \mathbb{H}^{2}(\Omega)$ which is the harmonic extension of $\left.f\right|_{\partial \Omega}$. So it is the solution to

$$
\begin{cases}\Delta h(x, y)=0 & \text { for all } x, y \in \Omega, \\ h(x, y)=f(x, y) & \text { for all } x, y \in \partial \Omega .\end{cases}
$$

Now $\tilde{f}=f-h$ vanishes at the boundary $\partial \Omega$ (so this requires Dirichlet boundary conditions in scale space) and can serve as an initial condition for the heat equation on the bounded domain. A practical method for obtaining $h$ on an arbitrarily shaped domain, is suggested by Georgiev (2005) and a fast method on a rectangular domain is proposed by Averbuch et al. (1998). Now $f_{m n}(x, y)$ is obtained by expansion of

$\tilde{f}=\sum_{m, n \in \mathbb{N}}\left(f_{m n}, \tilde{f}\right)_{\mathbb{L}_{2}(\Omega)} f_{m n}$,

which effectively exploits the sine transform.

The (fractional) operators that will appear in the construction of a Gaussian scale space on the bounded domain can be expressed as

$$
\begin{aligned}
& |\Delta|^{k} f_{m n}=\left|\lambda_{m n}\right|^{k} f_{m n}, \\
& e^{-s|\Delta|} f_{m n}=e^{s \lambda_{m n}} f_{m n} .
\end{aligned}
$$


Fig. 3 From left to right plots of the graph of $\mathbf{x} \mapsto G(\mathbf{x}, \mathbf{y})$, isocontours $\left\{\mathbf{x} \in \mathbb{R}^{2} \mid G(\mathbf{x}, \mathbf{y})=c\right\}$ for various $c>0$, and isocontours of its Harmonic conjugate $H(\mathbf{x}, \mathbf{y})=$ $\frac{-1}{2 \pi} \arg \left(\frac{\operatorname{sn}\left(x_{1}+i x_{2}, \tilde{k}\right)-\operatorname{sn}\left(y_{1}+i y_{2}, \tilde{k}\right)}{\tilde{s n}\left(x_{1}+i x_{2} \tilde{k}\right)-\operatorname{sn}\left(y_{1}+i y_{2}, \tilde{k}\right)}\right)$; $x_{1}$ runs along the horizontal axis whereas $x_{2}$ runs along the vertical axis. In the upper row $\left(y_{1}, y_{2}\right)=(0, \pi)$, and in the bottom row $\left(y_{1}, y_{2}\right)=(1,0.4)$. To keep $\log (z)=\log (|z|)+i \arg (z)=$ $\int_{1}^{z} \frac{1}{\xi} \mathrm{d} \xi$ single valued, we apply a branch-cut on the negative real axis. The thick lines in the $x_{1} x_{2}$-plane are mapped to the negative real axis, so here the graph of $H(\cdot, \mathbf{y})$ is discontinuous (the graph has a jump)
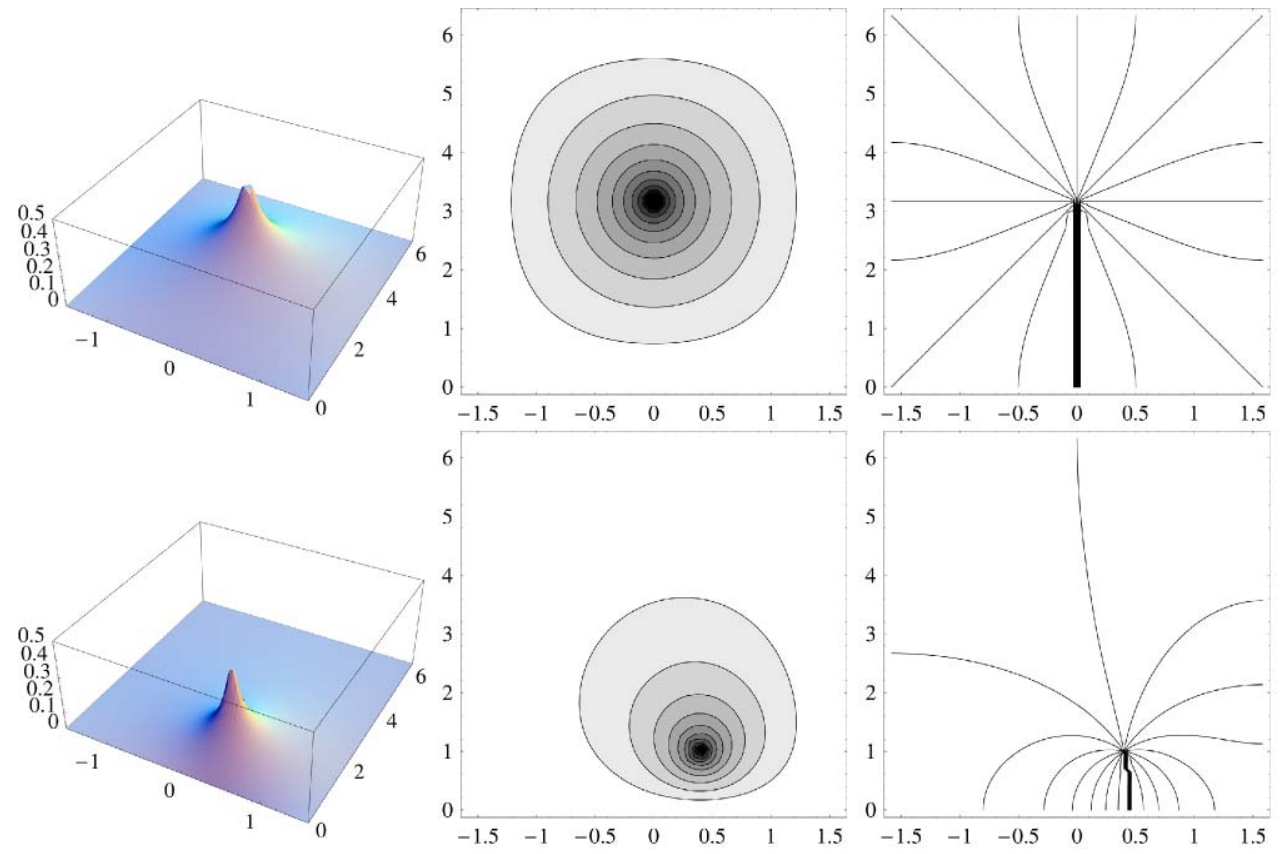

We also note that the $\kappa_{p}$ filters, defined in (8), are readily obtained by application of the following identity

$$
\left(I+\gamma^{2 k}|\Delta|^{k}\right)^{-1} f_{m n}=\frac{1}{1+\gamma^{2 k}\left|\lambda_{m n}\right|^{k}} f_{m n} .
$$

Consider the Gaussian scale space representation ${ }^{1}$ on bounded domain $\Omega$ (see Duits et al. 2003)

$u_{\tilde{f}}^{\Omega}(x, y, s)=\sum_{m, n \in \mathbb{N}} e^{s \lambda_{m n}}\left(f_{m n}, \tilde{f}\right)_{\mathbb{L}_{2}(\Omega)} f_{m n}(x, y)$

where the scale parameter $s \in \mathbb{R}^{+}$. It is the unique solution to

$$
\left\{\begin{array}{l}
\frac{\partial u}{\partial s}=\Delta u \\
\left.u(\cdot, s)\right|_{\partial \Omega}=0 \quad \text { for all } s>0 \\
u(\cdot, 0)=\tilde{f} .
\end{array}\right.
$$

We note that by straight forward computation one has

$$
\begin{aligned}
\Delta u_{\tilde{f}}^{\Omega}(x, y, s) & =\sum_{m, n \in \mathbb{N}} e^{s \lambda_{m n}}\left(f_{m n}, \tilde{f}\right)_{\mathbb{L}_{2}(\Omega)} \Delta f_{m n}(x, y) \\
& =\sum_{m, n \in \mathbb{N}} \lambda_{m n} e^{s \lambda_{m n}}\left(f_{m n}, \tilde{f}\right)_{\mathbb{L}_{2}(\Omega)} f_{m n}(x, y) \\
& =\partial_{s} \sum_{m, n \in \mathbb{N}} e^{s \lambda_{m n}}\left(f_{m n}, \tilde{f}\right)_{\mathbb{L}_{2}(\Omega)} f_{m n}(x, y) \\
& =\partial_{s} u_{\tilde{f}}^{\Omega}(x, y, s) .
\end{aligned}
$$

\footnotetext{
${ }^{1}$ The framework in this paper is readily generalized to $\alpha$-scale spaces in general (see e.g. Duits et al. 2003) by replacing $\left(-\lambda_{m n}\right)$ by $\left(-\lambda_{m n}\right)^{2 \alpha}$.
}

The filter $\phi_{p}$ that measures differential structure present in the scale space representation $u_{\tilde{f}}^{\Omega}$ of $\tilde{f}$ at a point $p$ with coordinates $\left(x_{p}, y_{p}, s_{p}\right)$, such that

$\left(D^{\mathbf{n}_{p}} u_{\tilde{f}}^{\Omega}\right)\left(x_{p}, y_{p}, s_{p}\right)=\left(\phi_{p}, \tilde{f}\right)_{\mathbb{L}_{2}(\Omega)}$,

is given by (writing multi-index $\left.\mathbf{n}_{p}=\left(n_{p}^{1}, n_{p}^{2}\right)\right)$

$$
\begin{aligned}
& \phi_{p}(x, y) \\
& \quad=\sum_{m, n \in \mathbb{N}} e^{s_{p} \lambda_{m n}}\left(D^{\mathbf{n}_{p}} f_{m n}\right)\left(x_{p}, y_{p}\right) f_{m n}(x, y),
\end{aligned}
$$

where we note that $\frac{\mathrm{d}}{\mathrm{d} x} \sin (x)=\cos (x)=\sin \left(x+\frac{\pi}{2}\right)$ and

$$
\begin{aligned}
\left(D^{\mathbf{n}_{p}} f_{m n}\right)\left(x_{p}, y_{p}\right)= & \sqrt{\frac{1}{a b}}\left(\frac{m \pi}{b}\right)^{n_{p}^{2}}\left(\frac{n \pi}{a}\right)^{n_{p}^{1}} \\
& \times \sin \left(\frac{m \pi y_{p}}{b}+\frac{\pi}{2} n_{p}^{2}\right) \\
& \times \sin \left(\frac{n \pi x_{p}}{a}+\frac{\pi}{2} n_{p}^{1}\right),
\end{aligned}
$$

$\mathbf{x}=(x, y) \in \Omega, \mathbf{x}_{p}=\left(x_{p}, y_{p}\right) \in \Omega$ and $\mathbf{n}_{p}=\left(n_{p}^{1}, n_{p}^{2}\right) \in$ $\mathbb{N} \times \mathbb{N}$. Here we use notation $\phi_{p}$ for the filters that measure features of the type presented in (27). However, we stress that this is just one particular case of the filters $\psi_{p}$ that are used to measure the general features, cf. (2).

\subsection{The Solution to the Reconstruction Problem}

Now that we have constructed a scale space on the bounded domain and shown how to measure its differential structure 
we can express the solution to the reconstruction problem (recall (7) and (8)) in terms of eigenfunctions and eigenvalues of the Laplace operator. To this end we recall that $V=\operatorname{span}\left\{\kappa_{q} \mid q \in 1, \ldots, P\right\}$ and we apply the orthogonal projection operator $\mathbb{P}_{V}: \mathbb{H}_{0}^{2 k, \gamma}(\Omega) \rightarrow V$ to $\tilde{f}$ :

$$
\begin{aligned}
\mathbb{P}_{V} \tilde{f} & =\sum_{p, q=1}^{P} G^{p q}\left(\kappa_{p}, \tilde{f}\right)_{\mathbb{H}_{0}^{2 k^{2}}(\Omega)} \kappa_{q} \\
& =\sum_{p, q=1}^{P} G^{p q}\left(\phi_{p}, \tilde{f}\right)_{\mathbb{L}_{2}(\Omega)} \kappa_{q} \\
& =\sum_{p, q=1}^{P} G^{p q} c_{p}(\tilde{f}) \kappa_{q},
\end{aligned}
$$

where $G^{p q}$ are components of the inverse of the Gram matrix. This implies $G^{p r} G_{r q}=\delta_{q}^{p}$, with $G_{p q}=\left(\kappa_{p}\right.$, $\left.\kappa_{q}\right)_{\mathbb{H}_{0}^{2 k, \gamma}(\Omega)}$. The filters $\kappa_{p}$ satisfy

$$
\begin{aligned}
\kappa_{p}(x, y)= & \sum_{m, n \in \mathbb{N}} \frac{e^{s_{p} \lambda_{m n}}}{1+\gamma^{2 k}\left|\lambda_{m n}\right|^{k}} \\
& \times\left(D^{\mathbf{n}_{p}} f_{m n}\right)\left(x_{p}, y_{p}\right) f_{m n}(x, y) .
\end{aligned}
$$

It can indeed be verified by direct computation that

$\mathbb{P}_{V}^{2}=\mathbb{P}_{V}, \quad \mathbb{P}_{V}^{*}=\mathbb{P}_{V}, \quad \mathcal{R}\left(\mathbb{P}_{V}\right)=V$,

so $\mathbb{P}_{V}$ is indeed the orthogonal projection onto $V$. The projection that is made explicit in (30) is, due to the Pythagoras theorem, the unique solution to the optimization problem

$$
\begin{aligned}
\underset{g \sim \tilde{f}}{\arg \min }\|g\|_{\mathbb{H}_{0}^{2 k, \gamma}(\Omega)}^{2}= & \underset{g \sim \tilde{f}}{\arg \min }\|\underbrace{g-\mathbb{P}_{V} \tilde{f}}_{\in V^{\perp}}\|_{\mathbb{H}_{0}^{2 k, \gamma}(\Omega)}^{2} \\
& +\|\underbrace{\mathbb{P}_{V} \tilde{f}}_{\in V}\|_{\mathbb{H}_{0}^{2 k, \gamma}(\Omega)}^{2},
\end{aligned}
$$

which was introduced in (7).

In order to compute the projection in (30) we apply a singular value decomposition (SVD) (Press et al. 1988, Chap. 2.9) to guarantee well-posedness of our algorithm. We briefly outline the application of the SVD to the projection in (30). Let the columns of the matrix $V$ be composed of the eigenvectors $\left\{\mathbf{v}_{i}\right\}_{i=1}^{P}$ of $G^{T} G$, where $G=\left[G_{p q}\right]_{p, q=1}^{P}$, with corresponding eigenvalues $\sigma_{i}^{2},\left(\sigma_{1}>\sigma_{2}>\ldots\right)$. We define $D=\operatorname{diag}\left\{\sigma_{i}\right\}$ and $U=G V D^{-1}$. This implies $U^{T}=U^{-1}$ and $\left(G^{T} G\right)^{-1} G^{T}=V D^{-1} U^{T}$, from which we deduce that

$\mathbb{P}_{V} \tilde{f}=\sum_{q=1}^{P} \sum_{i \in I}\left(\sum_{p=1}^{P} \frac{u_{i}^{p} c_{p}(\tilde{f})}{\sigma_{i}}\right) v_{i}^{q} \kappa_{q}$

with $\mathbf{u}_{i}$ the $i$ th column of $U$ and $u_{i}^{p}$ the $p$ th element of the vector $\mathbf{u}_{i}$. The set $I$ is defined as $I=\left\{i \in 1 \ldots P \mid \frac{\sigma_{i}}{\sigma_{1}} \geq\right.$ tolerance\}, thus directions corresponding to eigenvalues $\sigma_{i}$ such that $\frac{\sigma_{i}}{\sigma_{1}}$ is smaller than a given tolerance are removed from the projection.

\subsection{Neumann Boundary Conditions}

The axioms that naturally lead to $\alpha$-scale spaces (Duits et al. 2004) can not all be maintained in case of the bounded domain. One has to drop the axiom of translation invariance. This can be observed from Fig. 3: the Green's function of the Laplace operator on the bounded domain deforms when it is moved closer to the boundary. In order to maintain the other axioms such as gray value invariance and increase of entropy, Neumann boundary conditions should be chosen (Duits et al. 2003). Imposing zero Neumann boundary conditions coincides with the symmetric extension of the image at the boundaries and periodic boundary conditions on the extended domain. In this case the eigenvalues $\lambda_{m n}$ (18) are maintained, the eigenfunctions are given by

$$
\begin{aligned}
f_{m n}(x, y)= & \sqrt{\frac{1}{a b\left(1+\delta_{m 0}\right)\left(1+\delta_{n 0}\right)}} \\
& \times \cos \left(\frac{n \pi x}{a}\right) \cos \left(\frac{m \pi y}{b}\right),
\end{aligned}
$$

where $m, n \in \mathbb{N}$. We note that again:

$|\Delta|^{k} f_{m n}=\left|\lambda_{m n}\right|^{k} f_{m n}$.

When the eigenfunctions in (24) are replaced by the eigenfunctions in (35), (24) is the unique solution to

$\left\{\begin{array}{l}\frac{\partial u}{\partial s}=\Delta u, \\ \left.\frac{\partial u(\cdot, s)}{\partial \mathbf{n}}\right|_{\partial \Omega}=0 \quad \text { for all } s>0, \\ u(\cdot, 0)=\tilde{f},\end{array}\right.$

where $\mathbf{n}$ is the outward normal of the image boundary $\partial \Omega$.

When Neumann boundary conditions other than zero are required, we proceed in a similar fashion as proposed in Sect. 2.2. In this case, however, we have to take care that Green's second identity,

$\int_{\Omega} h \Delta f-f \Delta h \mathrm{~d} \mathbf{x}=\int_{\partial \Omega} h \frac{\partial f}{\partial \mathbf{n}}-f \frac{\partial h}{\partial \mathbf{n}} \mathrm{d} \sigma(\mathbf{x})$

with $\mathrm{d} \sigma(\mathbf{x})$ a boundary measure and $\mathbf{x} \in \Omega$, is not violated. As a consequence, we can not find an $h \in \mathbb{H}^{2}(\Omega)$ such that

$\left\{\begin{array}{l}\Delta h=0 \\ \left.\frac{\partial h}{\partial \mathbf{n}}\right|_{\partial \Omega}=\frac{\partial f}{\partial \mathbf{n}}\end{array}\right.$

holds, since

$\int_{\Omega} \Delta h \mathrm{~d} \mathbf{x}=\int_{\partial \Omega} \frac{\partial h}{\partial \mathbf{n}} \mathrm{d} \sigma(\mathbf{x})=\int_{\partial \Omega} \frac{\partial f}{\partial \mathbf{n}} \mathrm{d} \sigma(\mathbf{x})$. 
In order to solve this problem we introduce a constant source term in (39) and find instead an $h$ such that

$$
\left\{\begin{array}{l}
\Delta h=K, \\
\left.\frac{\partial h}{\partial \mathbf{n}}\right|_{\partial \Omega}=\frac{\partial f}{\partial \mathbf{n}},
\end{array}\right.
$$

with $K=\frac{1}{|\Omega|} \int_{\partial \Omega} \frac{\partial f}{\partial \mathbf{n}} \mathrm{d} \sigma(\mathbf{x})$ and $|\Omega|$ the area of the domain. An efficient method to solve $h$ from (41) based on the method by Averbuch et al. (1998) can be found in Yamatani and Saito (2006). Now $\tilde{f}=f-h$ has zero normal derivatives at the boundary $\partial \Omega$ and can serve as an initial condition for the heat equation that is presented in (37).

\subsection{Singular Points}

The theory presented in the previous subsections is applicable to generic linear features, cf. (2). In Sect. 4 we will apply our reconstruction method to image reconstruction from differential properties of so called singular points of a scale space representation of an image. Therefore we will briefly summarize how to obtain the locations of these points. A non-Morse critical point of a scale space representation of an image, to which we will henceforth refer to as a singular point, is defined as follows:

Definition 2 (Singular Point) A singular point $(x, y, s) \in$ $\mathbb{R}^{2} \times \mathbb{R}^{+}$of a scale space representation $u_{f}^{\Omega}(x, y, s)$ of the image $f$ is defined by the following equations, in which $\nabla$ denotes the spatial gradient operator:

$$
\left\{\begin{array}{l}
\nabla u_{f}^{\Omega}(x, y, s)=0, \\
\operatorname{det} \nabla \nabla^{T} u_{f}^{\Omega}(x, y, s)=0 .
\end{array}\right.
$$

See Damon (1995), Florack and Kuijper (2000) for further details, and Gilmore (1993) for a general introduction to catastrophe theory. Figure 4 illustrates the set of singular points for a typical image. Solutions of (42) can be found by a zero-crossings method (Lorensen and Cline 1987; Kanters et al. 2004). Given an initial approximate location of a singular point, $\left(x_{a}, y_{a}, s_{a}\right)$, we can refine its position in scale space to a corrected position, $\left(x_{c}, y_{c}, s_{c}\right)$, by calculating $\left(x_{c}, y_{c}, s_{c}\right)=\left(x_{a}+\delta x, y_{a}+\delta y, s_{a}+\delta s\right)$, where

$$
\begin{aligned}
{\left[\begin{array}{l}
\delta x \\
\delta y \\
\delta s
\end{array}\right]=} & \left(\mathbf{M}\left(x_{a}, y_{a}, s_{a}\right)\right)^{-1} \\
& \times\left[\begin{array}{c}
\mathbf{g}\left(x_{a}, y_{a}, s_{a}\right) \\
\left(\operatorname{det} \nabla \nabla^{T} u_{f}^{\Omega}\right)\left(x_{a}, y_{a}, s_{a}\right)
\end{array}\right],
\end{aligned}
$$

and where

$$
\begin{aligned}
& \mathbf{M}=\left[\begin{array}{cc}
\nabla \nabla^{T} u_{f}^{\Omega} & \mathbf{w} \\
\mathbf{z}^{T} & c
\end{array}\right], \\
& \mathbf{g}(x, y, s)=\nabla u_{f}^{\Omega}(x, y, s), \quad \mathbf{w}=\partial_{s} \mathbf{g},
\end{aligned}
$$

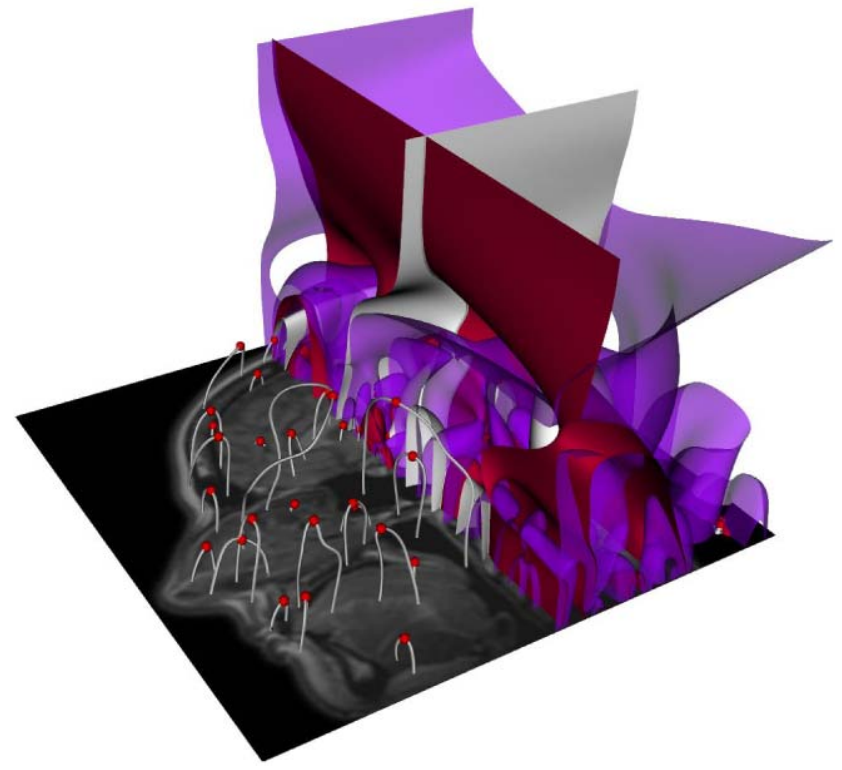

Fig. 4 A visualization of the "deep structure" of a Gaussian scale space representation of an image. The gray paths represent critical paths (paths of vanishing gradient), a red ball shows the location of a singular point on a critical path. The surfaces show the iso-surfaces given by $\frac{\partial u_{f}^{\Omega}(x, y, s)}{\partial x}=0, \frac{\partial u_{f}^{\Omega}(x, y, s)}{\partial y}=0$ and $\operatorname{det} \nabla \nabla^{T} u_{f}^{\Omega}(x, y, s)=0$, which are used in the calculation of the position of a singular point. Critical paths are found by intersecting the surfaces that satisfy $\frac{\partial u_{f}^{\Omega}(x, y, s)}{\partial x}=0$ and $\frac{\partial u_{f}^{\Omega}(x, y, s)}{\partial y}=0$. Singular points are found by intersecting the critical paths with the surface that satisfies $\left(\operatorname{det} \nabla \nabla^{T} u_{f}^{\Omega}\right)(x, y, s)=0$

$\mathbf{z}(x, y, s)=\nabla \operatorname{det} \nabla \nabla^{T} u_{f}^{\Omega}(x, y, s)$,

$c(x, y, s)=\partial_{s} \operatorname{det} \nabla \nabla^{T} u_{f}^{\Omega}(x, y, s)$.

Notice that all derivatives are taken in the point $\left(x_{a}, y_{a}, s_{a}\right)$. This procedure is repeated in order to obtain a more accurate location of a singular point. For further details we refer to Florack and Kuijper (2000), Platel (2007). In general, images are not determined by their singular points. Consider for example the class of images

$f(x, y)=f_{m n}(x, y)$,

whose bounded domain scale space representations are given by

$u_{f}^{\Omega}(x, y, s)=e^{s \lambda_{m n}} f_{m n}(x, y)$.

These scale space representations do not contain any singular points. However, scale space representations of natural images do contain singular points attached to generic topological transitions (Damon 1995). If one endows these points with suitable attributes one can obtain a reconstruction that is visually close to the initial image (Janssen et al. 2006). 


\section{Implementation}

The implementation of the reconstruction method that was presented in a continuous Hilbert space framework is completely performed in a discrete framework in order to avoid approximation and truncation errors due to sampling. Here we shall make use of a discrete sine transform (rather than truncated Fourier series) and its inverse, which are exact on the grid.

First we introduce the discrete sine transform $\mathcal{F}_{S}$ : $l_{2}\left(I_{N}^{\mathcal{D}}\right) \rightarrow l_{2}\left(I_{N}^{\mathcal{D}}\right)$ on a rectangular domain $I_{N}^{\mathcal{D}}=\{1, \ldots$, $N-1\} \times\{1, \ldots, M-1\}$

$$
\begin{aligned}
& \left(\mathcal{F}_{S} f\right)(u, v) \\
& =-\frac{2}{\sqrt{M N}} \sum_{i=1}^{M-1} \sum_{j=1}^{N-1} \underbrace{\sin \left(\frac{i u \pi}{M}\right) \sin \left(\frac{j v \pi}{N}\right)}_{\left(\varphi_{i} \otimes \varphi_{j}\right)(u, v)} f(i, j),
\end{aligned}
$$

with $(u, v) \in I_{N}^{\mathcal{D}}$. Notice that this unitary transform is its own inverse and that

$\left(\varphi_{i}, \varphi_{j}\right)_{\mathrm{t}_{2}\left(I_{N}^{\mathcal{D}}\right)}=\delta_{i j}$,

so $\left\{\varphi_{i} \otimes \varphi_{j} \mid \begin{array}{c}i=1, \ldots, M-1 \\ j=1, \ldots, N-1\end{array}\right\}$ forms an orthonormal basis in $l_{2}\left(I_{N}^{\mathcal{D}}\right)$.

The Gaussian scale space representation $u_{f}^{I_{N}^{\mathcal{D}}}(i, j, s)$ of an image $f \in l_{2}\left(I_{N}^{\mathcal{D}}\right)$ introduced in the continuous domain in (24) now reads

$$
\begin{aligned}
u_{f}^{I_{N}^{\mathcal{D}}}(i, j, s)= & \left(e^{s \Delta} f\right)(i, j) \\
= & -\frac{2}{\sqrt{M N}} \sum_{u=1}^{M-1} \sum_{v=1}^{N-1} \hat{f}(u, v) e^{-s\left(\frac{u^{2}}{M^{2}}+\frac{v^{2}}{N^{2}}\right) \pi^{2}} \\
& \times\left(\varphi_{u} \otimes \varphi_{v}\right)(i, j)
\end{aligned}
$$

where $\hat{f}(u, v)=\left(\mathcal{F}_{S} f\right)(u, v)$. Differential structure of or$\operatorname{der} \mathbf{n}_{p}=\left(n_{p}^{1}, n_{p}^{2}\right) \in \mathbb{N} \times \mathbb{N}$ at a certain position $\left(i_{p}, j_{p}\right) \in$ $I_{N}^{\mathcal{D}}$ and at scale $s_{p} \in \mathbb{R}^{+}$is measured by

$$
\begin{aligned}
\left(D^{\mathbf{n}_{p}} u_{f}^{I_{N}^{\mathcal{D}}}\right)\left(i_{p}, j_{p}, s_{p}\right) \\
=-\frac{2}{\sqrt{M N}} \sum_{u=1}^{M-1} \sum_{v=1}^{N-1} \hat{f}(u, v) e^{-s_{p}\left(\frac{u^{2}}{M^{2}}+\frac{v^{2}}{N^{2}}\right) \pi^{2}} \\
\quad \times\left(\frac{u \pi}{M}\right)^{n_{p}^{1}}\left(\frac{v \pi}{N}\right)^{n_{p}^{2}} \sin \left(\frac{i_{p} u \pi}{M}+\frac{\pi}{2} n_{p}^{1}\right) \\
\quad \times \sin \left(\frac{j_{p} v \pi}{N}+\frac{\pi}{2} n_{p}^{2}\right) .
\end{aligned}
$$

The filters $\phi_{p}$, with $p=\left(i_{p}, j_{p}, s_{p}, \mathbf{n}_{p}\right)$ a multi-index, are given by

$$
\begin{aligned}
\phi_{p}(i, j, s) & \\
= & -\frac{2}{\sqrt{M N}} \sum_{u=1}^{M-1} \sum_{v=1}^{N-1} e^{-s_{p}\left(\frac{u^{2}}{M^{2}}+\frac{v^{2}}{N^{2}}\right) \pi^{2}}\left(\varphi_{u} \otimes \varphi_{v}\right)(i, j) \\
& \times\left(\frac{u \pi}{M}\right)^{n_{p}^{1}}\left(\frac{v \pi}{N}\right)^{n_{p}^{2}} \sin \left(\frac{i_{p} u \pi}{M}+\frac{\pi}{2} n_{p}^{1}\right) \\
& \times \sin \left(\frac{j_{p} v \pi}{N}+\frac{\pi}{2} n_{p}^{2}\right)
\end{aligned}
$$

and the filters $\kappa_{p}$ corresponding to $\phi_{p}$ read

$\kappa_{p}(i, j, s)$

$$
\begin{aligned}
= & -\frac{2}{\sqrt{M N}} \sum_{u=1}^{M-1} \sum_{v=1}^{N-1} \frac{e^{-s_{p}\left(\frac{u^{2}}{M^{2}}+\frac{v^{2}}{N^{2}}\right) \pi^{2}}}{1+(\pi \gamma)^{2 k}\left(\frac{u^{2}}{M^{2}}+\frac{v^{2}}{N^{2}}\right)^{k}} \\
& \times\left(\varphi_{u} \otimes \varphi_{v}\right)(i, j)\left(\frac{u \pi}{M}\right)^{n_{p}^{1}}\left(\frac{v \pi}{N}\right)^{n_{p}^{2}} \\
& \times \sin \left(\frac{i_{p} u \pi}{M}+\frac{\pi}{2} n_{p}^{1}\right) \sin \left(\frac{j_{p} v \pi}{N}+\frac{\pi}{2} n_{p}^{2}\right) .
\end{aligned}
$$

An element $G_{p q}=\left(\phi_{p}, \phi_{q}\right)_{l_{2}\left(I_{N}^{\mathcal{D}}\right)}$ of the Gram matrix can, because of the orthonormality of the transform, be expressed in just a double sum,

$$
\begin{aligned}
G_{p q}= & -\frac{2}{\sqrt{M N}} \sum_{u=1}^{M-1} \sum_{v=1}^{N-1} \frac{e^{-\left(s_{p}+s_{q}\right)\left(\frac{u^{2}}{M^{2}}+\frac{v^{2}}{N^{2}}\right) \pi^{2}}}{1+(\pi \gamma)^{2 k}\left(\frac{u^{2}}{M^{2}}+\frac{v^{2}}{N^{2}}\right)^{k}} \\
& \times\left(\frac{u \pi}{M}\right)^{n_{p}^{1}}\left(\frac{v \pi}{N}\right)^{n_{p}^{2}} \sin \left(\frac{i_{p} u \pi}{M}+\frac{\pi}{2} n_{p}^{1}\right) \\
& \times \sin \left(\frac{j_{p} v \pi}{N}+\frac{\pi}{2} n_{p}^{2}\right)\left(\frac{u \pi}{M}\right)^{n_{q}^{1}}\left(\frac{v \pi}{N}\right)^{n_{q}^{2}} \\
& \times \sin \left(\frac{i_{q} u \pi}{M}+\frac{\pi}{2} n_{q}^{1}\right) \sin \left(\frac{j_{q} v \pi}{N}+\frac{\pi}{2} n_{q}^{2}\right) .
\end{aligned}
$$

In order to gain accuracy we implement (55) by summing in the reverse direction and multiplying by $\gamma^{2 k}$. Then we compute

$\tilde{g}=\sum_{p, q=1}^{P} G^{p q} \gamma^{2 k} c_{p}(f) \phi_{q}$

and find the reconstructed image $g$ by filtering $\tilde{g}$ by a discrete version of the 2D Butterworth filter of order $2 k$ and with cut-off frequency $\omega_{0}=\frac{1}{\gamma}$.

The implementation was written using the sine transform as defined in (49) where we already explicitly mentioned that the transform can be written as

$$
\begin{aligned}
& \left(\mathcal{F}_{S} f\right)(u, v)= \\
& -\frac{2}{\sqrt{M N}} \sum_{i=1}^{M-1} \sum_{j=1}^{N-1}\left(\varphi_{i} \otimes \varphi_{j}\right)(u, v) f(i, j) .
\end{aligned}
$$


Now we define the cosine transform, $\mathcal{F}_{S}: l_{2}\left(I_{N}^{\mathcal{N}}\right) \rightarrow$ $l_{2}\left(I_{N}^{\mathcal{N}}\right)$, on a rectangular domain, $I_{N}^{\mathcal{N}}=\{0, \ldots, N-1\} \times$ $\{0, \ldots, M-1\}$, in a similar manner

$$
\left(\mathcal{F}_{C} f\right)(u, v)=\sum_{i=0}^{M-1} \sum_{j=0}^{N-1}\left(\tilde{\varphi}_{i} \otimes \tilde{\varphi}_{j}\right)(u, v) f(i, j),
$$

where

$$
\begin{aligned}
\left(\tilde{\varphi_{i}} \otimes \tilde{\varphi_{j}}\right)(u, v)= & \cos \left(\frac{\pi\left(i+\frac{1}{2}\right) u}{M}\right) \sqrt{\frac{2-\delta_{u 0}}{M}} \\
& \times \cos \left(\frac{\pi\left(j+\frac{1}{2}\right) v}{M}\right) \sqrt{\frac{2-\delta_{v 0}}{N}}
\end{aligned}
$$

and $(u, v) \in I_{N}^{\mathcal{N}}$. These cosine basis functions $\left\{\tilde{\varphi}_{i} \otimes \tilde{\varphi}_{j} \mid \begin{array}{c}i=0, \ldots, M-1 \\ j=0, \ldots, N-1\end{array}\right\}$ form an orthogonal basis in $l_{2}\left(I_{N}^{\mathcal{N}}\right)$ and can thus be used to transform the reconstruction method that was explicitly presented for the Dirichlet case into a reconstruction method based on Neumann boundary conditions.

\section{Experiments}

We evaluate the reconstruction method by applying it to the problem that was presented in the introduction. The upper row of Fig. 5 shows from left to right: the image from which the features were extracted, a reconstruction by the unbounded domain method (Janssen et al. 2006) (parameters: $\gamma=50, k=1$ ), and a reconstruction by the newly introduced bounded domain method using Dirichlet boundary conditions (parameters: $\gamma=50, k=1$ ). Features that were used are up to second order derivatives measured at the singular points (see Sect. 2.5) of the scale space representation of the original image $f$. One can clearly see that the structure that is missing in the middle image (cf. Fig. 1) does appear when the bounded domain method is used (top-right image in Fig. 5).

The visual quality of the reconstruction in the top-right image in Fig. 5 is not appealing. In order to obtain a more appealing reconstruction one could, like in our previous work on image reconstruction (Janssen et al. 2006), endow the feature points with higher order differential structure. Another possibility is to select a different set of features. We proceed with the latter approach.

Singular points of a scale space representation of an image tend to catch blob-like structures, whereas singular points of the scale space representation of the Laplacian of an image (henceforth called Laplacian singular points) are more likely to catch information about edges and ridges in the image. This happens because the Laplacian tends to act as an edge detector. Furthermore, the number of singular points that manifest themselves in the scale space of an image is much smaller than the number of Laplacian singular points of an image. If more information about the image is used by the reconstruction algorithm, e.g. in the form of more features, one can expect to obtain a more appealing reconstruction. This motivates us to reconstruct from the properties of Laplacian singular points instead of singular points.

The bottom row of Fig. 5 shows reconstructions from up to second order differential structure obtained from the scale space representation of $f$, evaluated at the locations of the singular points of the scale space representation of the Laplacian of $f$. On the left the unbounded domain method was used with $\gamma=100$ and $k=1$, this leads to a reconstructed signal that has "spilled" too much over the border of the image and therefore is not as sharp as the reconstruction obtained by our newly proposed method using Dirichlet boundary conditions (parameters: $\gamma=100$ and $k=1$ ). Due to this spilling, the Gram matrix of the unbounded domain reconstruction method is harder to invert since basis functions start to become more and more dependent. This problem gets worse when $\gamma$ increases. Our bounded domain method is immune to this problem as long as the parameter $k$ is not chosen too high.

In order to quantify the observation that the Gram matrix is harder to invert when $\gamma$ gets larger, we applied both the reconstruction method presented in this paper and the unbounded domain method (Janssen et al. 2006) to the reconstruction from up to second order derivatives measured at the singular points of the scale space representation of Lena's eye. Lena's eye is the left-most image in Fig. 6 and has dimensions of $64 \times 64$ pixels. We fixed $k=1$ and set the tolerance of the singular value decomposition (SVD) algorithm that was used to compute the inverse of the Gram matrix (see Sect. 2.3, (34)) to $10^{-7}$. The percentage of features that were removed by the SVD algorithm as a function of $\gamma$ is displayed in Fig. 7. From this figure we can observe the unbounded domain method breaks down for $\gamma \gg 0$, whereas the method that is presented in this paper is much more robust. Even for $\gamma \gg 0$ barely any features are removed by the thresholding step in the SVD algorithm.

If the order of the Sobolev space $k$ is chosen too high, our method also breaks down. Figure 8 shows several reconstructions from up to second order differential structure taken at the locations of the singular points of Brabara's face using Dirichlet boundary conditions. The image from which the features were obtained is the right-most image in Fig. 6 and has dimensions of $128 \times 128$ pixels. From top to bottom the parameter $k=\{0.5+\epsilon, 1.0,1.5,2.0,2.5\}$ (introduced in (4)), which controls the order of the Sobolev space we are projecting in is varied. From left to right the parameter $\gamma=\{1,4,16,64,256,1024\}$ (introduced in (6)), which controls the importance of smoothness is varied. One 


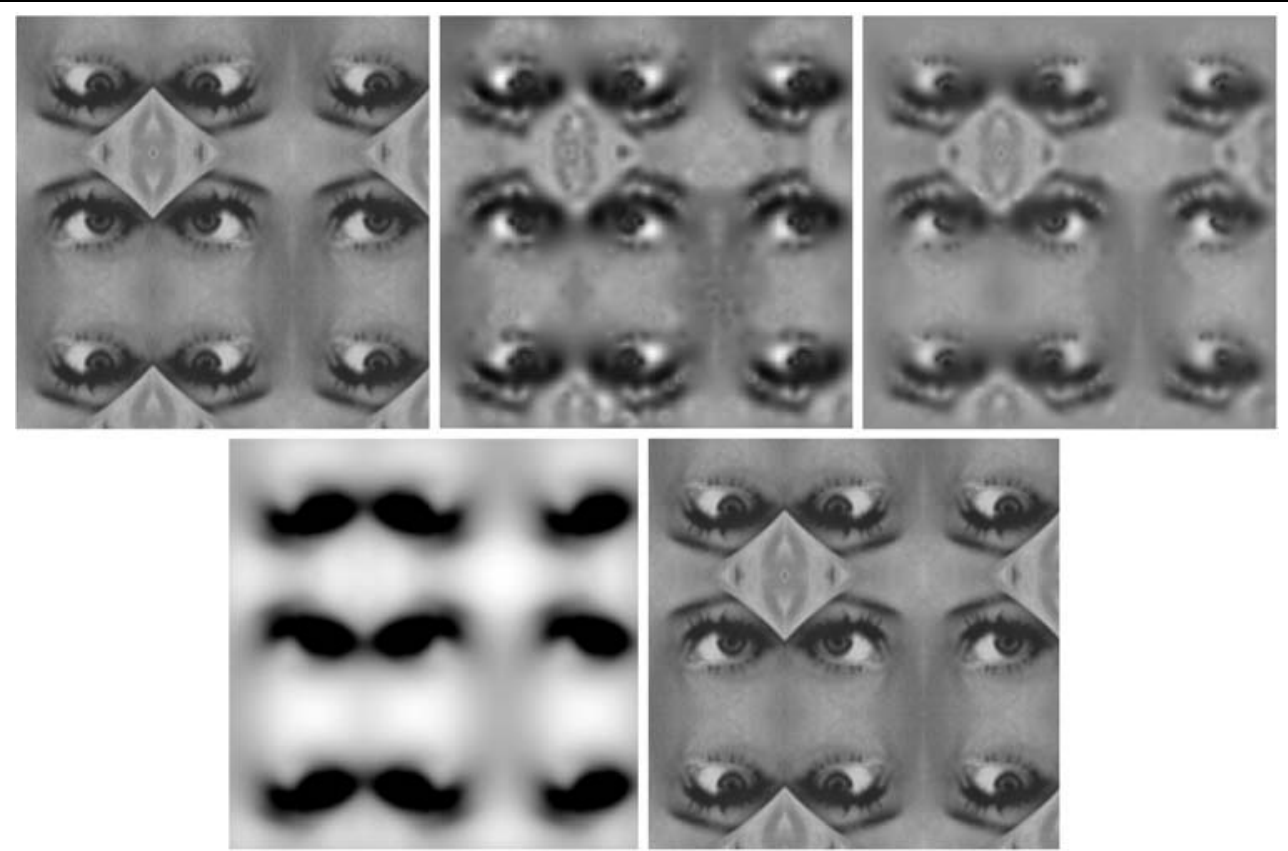

Fig. 5 Top left: The image $f$ from which the features were extracted. Top center and right: reconstruction from second order structure of the singular points of $f$ using the unbounded domain method (Janssen et al. 2006) (parameters: $\gamma=50, k=1$ ) and the bounded domain method (parameters: $\gamma=50, k=1$ ). Bottom row: unbounded domain (left) and bounded domain (right) reconstruction from up to second order differential structure obtained from the scale space represen- tation of $f$, evaluated at the locations of the singular points of the Laplacian of $f$. The parameters for the reconstruction algorithm are $k=1$ and $\gamma=100$. The latter value of $\gamma$ results in a blurred reconstruction for the unbounded domain method, whereas the bounded domain method does produce a visually appealing reconstruction (lower right) of the image that is shown in the upper left corner of this figure
Fig. 6 Input images that were used in the experiments. The left image shows Lena's eye, which is an image patch of $64 \times 64$ pixels. On the right Barbara's face is shown. This is an image patch of $128 \times 128$ pixels
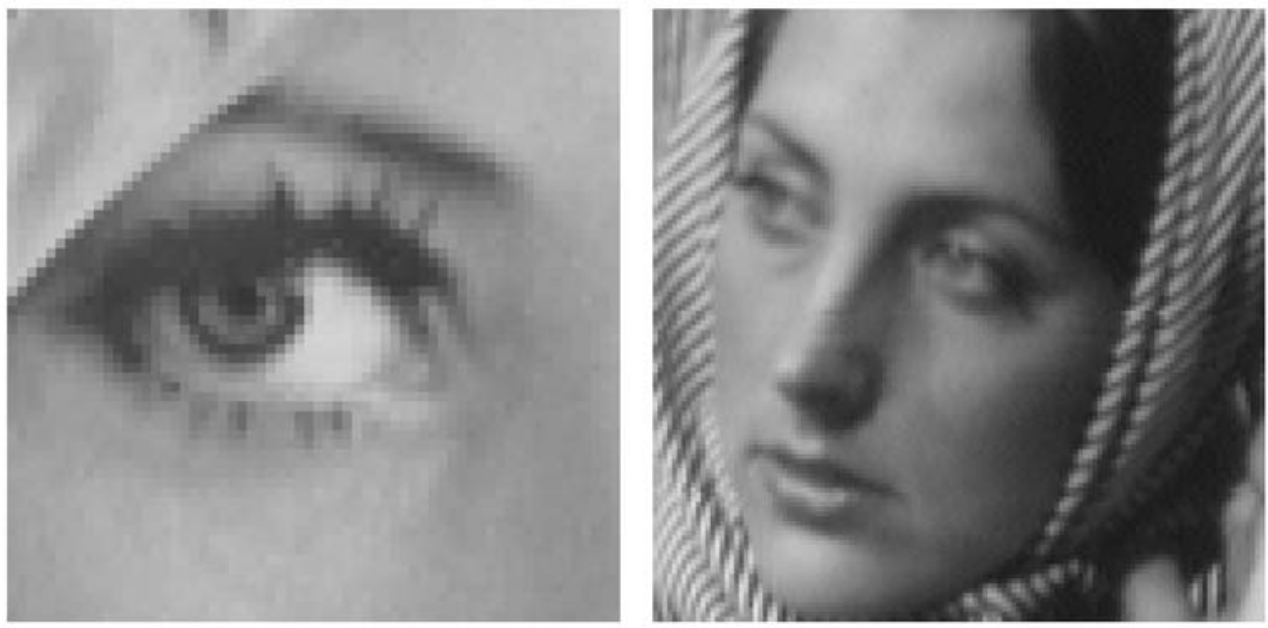

can clearly see the effect of the parameters. We will interpret the results in terms of the low-pass Butterworth filter introduced in (11). When $k$ is increased the order of the filter increases and consequently approximates the ideal low-pass filter more closely. In the bottom row of Fig. $8(k=2.5)$ the filter is too sharp (i.e. it approximates the ideal lowpass filter too closely) which results in a reconstruction that does not satisfy all features. This effect is even visible when $\gamma=1$. Increasing $\gamma$ corresponds to decreasing the cut-off frequency of the filter, which smooths the "bumpy" features that are most apparent in the top-most sub-images of Fig. 8. If $k=1$ increasing $\gamma$ does not cause problems for the inversion of the Gram matrix (and consequent loss of features). This can also be observed from Fig. 7. It does have a smoothing effect on the reconstructed image. Therefore it is preferred to use a $\gamma \gg 0$. 
Fig. 7 The percentage of features dropped by the singular value decomposition (SVD) algorithm that is used for the inversion of the Gram matrix as a function of $\gamma$. The $\gamma$ axis is sampled logarithmically. Features used are up to second order derivatives measured at the singular points of the scale space representation of Lena's eye (see Fig. 6). The SVD tolerance is set to $10^{-7}$ and $k=1$ for both the bounded domain and the unbounded domain method

Fig. 8 Reconstructions from second order differential structure taken at the locations of the singular points of the scale space representation of Brabara's face using Dirichlet boundary conditions. From top to bottom

$k=\{0.5+\epsilon, 1.0,1.5,2.0,2.5\}$ and from left to right $\gamma=\{1,4,16,64,256,1024\}$. The image from which the features were obtained is shown in Fig. 6
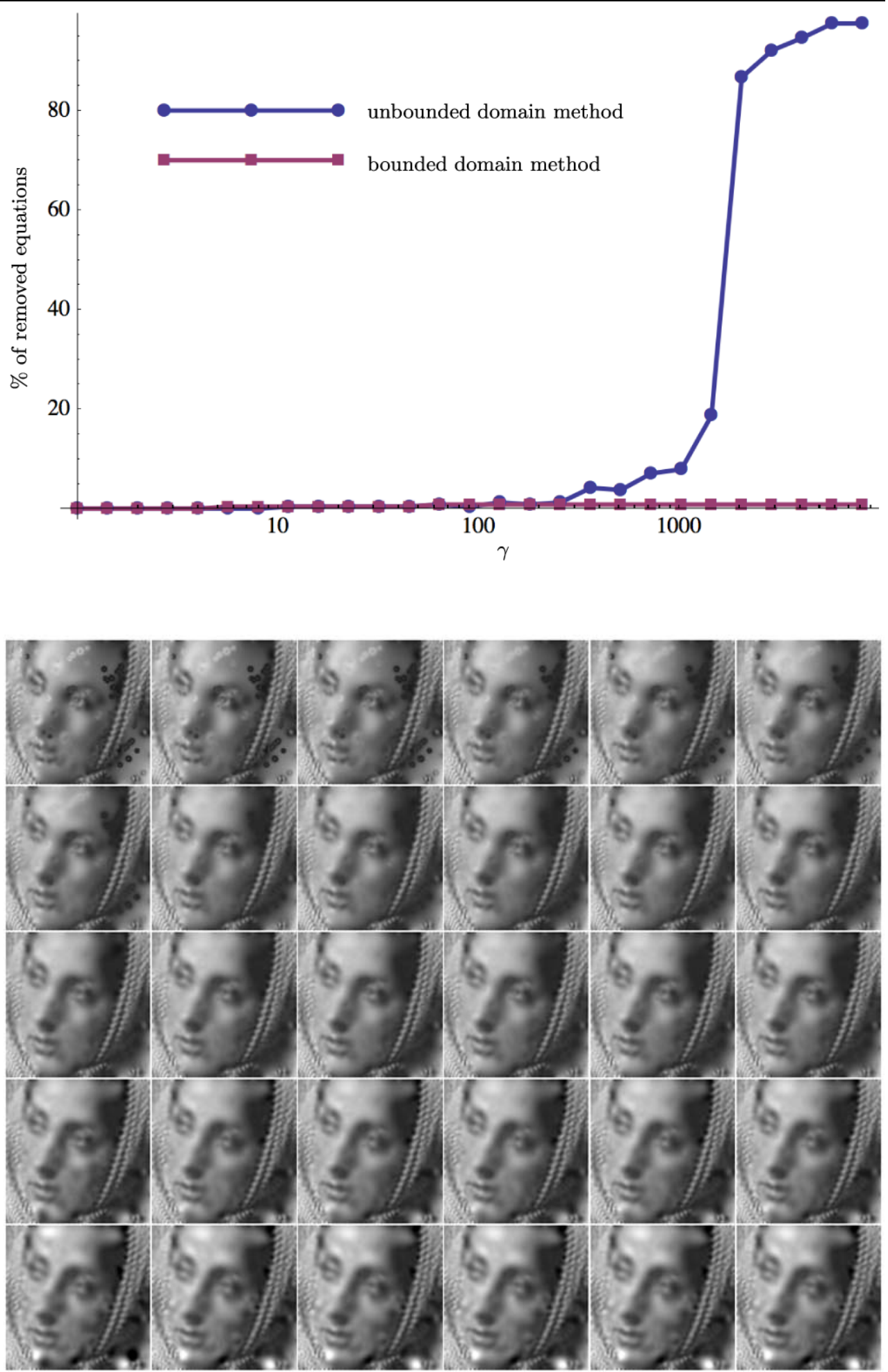

\section{Conclusion}

In previous work we considered the image as compactly supported in $\mathbb{L}_{2}\left(\mathbb{R}^{2}\right)$ and we used Sobolev norms on the unbounded domain including a smoothing parameter $\gamma>0$ to tune the smoothness of the reconstructed image. Due to the assumption of compact support of the original image components of the reconstructed image near the image boundary are too much penalized. Therefore we proposed to minimize Sobolev norms only on the actual image domain, yield- 
ing much better reconstructions (especially for $\gamma \gg 0$ ). We give a closed form expression for the Green's function of the Dirichlet operator in the spatial domain and put a relation between the fundamental solution of the Laplace operator on the unbounded domain and the Green's function on the bounded domain with Dirichlet conditions. Both feature extraction and the reconstruction method are formulated on the bounded domain in terms of the eigenfunctions and corresponding eigenvalues of the Laplace operator on the bounded domain: $\Delta: \mathbb{H}_{0}^{2}(\Omega) \rightarrow \mathbb{L}_{2}(\Omega)$. By changing the eigenfunctions the Dirichlet boundary conditions can be interchanged with Neumann boundary conditions. The implementation is done completely in the discrete domain and is exact on the grid, avoiding truncation or approximation errors. This is achieved by making use of fast discrete sine or discrete cosine transforms.

We also showed an interpretation for the parameter $\gamma$ and the order of the Sobolev space $k$ in terms of filtering by the classical Butterworth filter. In future work we plan to exploit this interpretation by automatically selecting the order of the Sobolev space.

Acknowledgements The authors would like to thank the anonymous referees whose comments and suggestions led to a significant improvement of the presentation and organization of this paper. The Netherlands Organisation for Scientific Research (NWO) is gratefully acknowledged for financial support. The image in Fig. 4 was provided by Dr. B. Platel, Eindhoven University of Technology, department of Biomedical Engineering.

Open Access This article is distributed under the terms of the Creative Commons Attribution Noncommercial License which permits any noncommercial use, distribution, and reproduction in any medium, provided the original author(s) and source are credited.

\section{Appendix: Closed from Expression of the Green's Function of the Dirichlet Operator on a Rectangle}

The Green's function $G: \Omega \times \Omega \rightarrow \mathbb{R}$ of the Dirichlet operator $\mathcal{D}$ (recall Definition 1) can be obtained by means of conformal mapping ${ }^{2}$. A visualization of the mappings used to arrive at the solution can be found in Fig. 9 .

To this end we first map the rectangle to the upper half space in the complex plane. By the Schwarz-Christoffel formula the derivative of the inverse of such a mapping is given by

$$
\begin{aligned}
\frac{\mathrm{d} z}{\mathrm{~d} w} & =-C \frac{1}{\tilde{k}}(w-1)^{-\frac{1}{2}}(w+1)^{-\frac{1}{2}}\left(w-\frac{1}{\tilde{k}}\right)^{-\frac{1}{2}}\left(w+\frac{1}{\tilde{k}}\right)^{-\frac{1}{2}} \\
& =C \frac{1}{\sqrt{1-w^{2}}} \frac{1}{\sqrt{1-\tilde{k}^{2} w^{2}}},
\end{aligned}
$$

${ }^{2}$ Our solution is a generalization of the solution derived by Boersma et al. (2002). where $C \in \mathbb{R}^{+}$and $w( \pm 1 / \tilde{k})= \pm a+i b$. As a result

$$
\begin{gathered}
z(w, \tilde{k})=C \int_{0}^{w} \frac{\mathrm{d} t}{\sqrt{1-t^{2}} \sqrt{1-\tilde{k}^{2} t^{2}}} \\
\Leftrightarrow \quad w(z)=\operatorname{sn}\left(\frac{z}{C}, \tilde{k}\right),
\end{gathered}
$$

where $s n$ denotes the Jacobi-elliptic function (Gradshteyn and Ryzhik 1994; Whittaker and Watson 1946, Chap. XXII). We have $\operatorname{sn}(0, \tilde{k})=0, \operatorname{sn}( \pm a, \tilde{k})= \pm 1, \operatorname{sn}( \pm a+i b, \tilde{k})=$ $\pm(1 / \tilde{k})$ and $\operatorname{sn}(i(b / 2), \tilde{k})=i / \sqrt{\tilde{k}}$, where the elliptic modulus $\tilde{k}$ is given by

$(b / a) z(1, \tilde{k})=z\left(1, \sqrt{1-\tilde{k}^{2}}\right)$.

We note that for every fraction $b / a \in \mathbb{R}^{+}$there is a unique $\tilde{k}$ that satisfies (62). For example, in case of a square $b / a=2$ we have $\tilde{k} \approx 0.1715728752$. The reader must be aware that $\tilde{k}$ is a function of the aspect ratio $b / a$.

For the moment we assume

$a=z(1, \tilde{k})$

and

$b=z\left(1, \sqrt{1-\tilde{k}^{2}}\right)$,

which implies $C=1$. The next step is to map the half plane onto the unit disk $\overline{B_{\mathbf{0}, 1}}=\left\{\mathbf{x} \in \mathbb{R}^{2} \mid\|\mathbf{x}\| \leq 1\right\}$. This is easily done by means of a linear fractional transform

$\chi(z)=\frac{z-\operatorname{sn}\left(y_{1}+i y_{2}, \tilde{k}\right)}{z-\overline{\operatorname{sn}\left(y_{1}+i y_{2}, \tilde{k}\right)}}$.

To this end we notice that $|\chi(0)|=1$ and that the mirrored points $\operatorname{sn}\left(y_{1}+i y_{2}, \tilde{k}\right)$ and $\overline{\operatorname{sn}\left(y_{1}+i y_{2}, \tilde{k}\right)}$ are mapped to the mirrored points $\chi\left(\operatorname{sn}\left(y_{1}+i y_{2}, \tilde{k}\right)\right)=0$ and $\chi\left(\overline{\operatorname{sn}\left(y_{1}+i y_{2}, \tilde{k}\right)}\right)=\infty$.

Now define $F: \mathbb{C} \rightarrow \mathbb{C}$ and $\mathbf{F}: \bar{\Omega} \rightarrow \overline{B_{\mathbf{0}, 1}}$ by

$F=\chi \circ \operatorname{sn}(\cdot, \tilde{k}), \quad$ i.e.

$F\left(x_{1}+i x_{2}\right)=\frac{\operatorname{sn}\left(x_{1}+i x_{2}, \tilde{k}\right)-\operatorname{sn}\left(y_{1}+i y_{2}, \tilde{k}\right)}{\operatorname{sn}\left(x_{1}+i x_{2}, \tilde{k}\right)-\overline{\operatorname{sn}\left(y_{1}+i y_{2}, \tilde{k}\right)}}$,

$\mathbf{F}\left(x_{1}, x_{2}\right)=\left(\operatorname{Re}\left(F\left(x_{1}+i x_{2}\right)\right), \operatorname{Im}\left(F\left(x_{1}+i x_{2}\right)\right)\right)^{T}$,

then $\mathbf{F}$ is a conformal mapping of $\bar{\Omega}$ onto $\overline{B_{\mathbf{0}, 1}}$ with $\mathbf{F}(\mathbf{y})=$ 0. As a result we have by the Cauchy-Riemann equations

$\Delta_{\mathbf{F}(\mathbf{x})}=\left|\mathbf{F}^{\prime}(\mathbf{x})\right|^{-1} \Delta_{\mathbf{x}}$, 

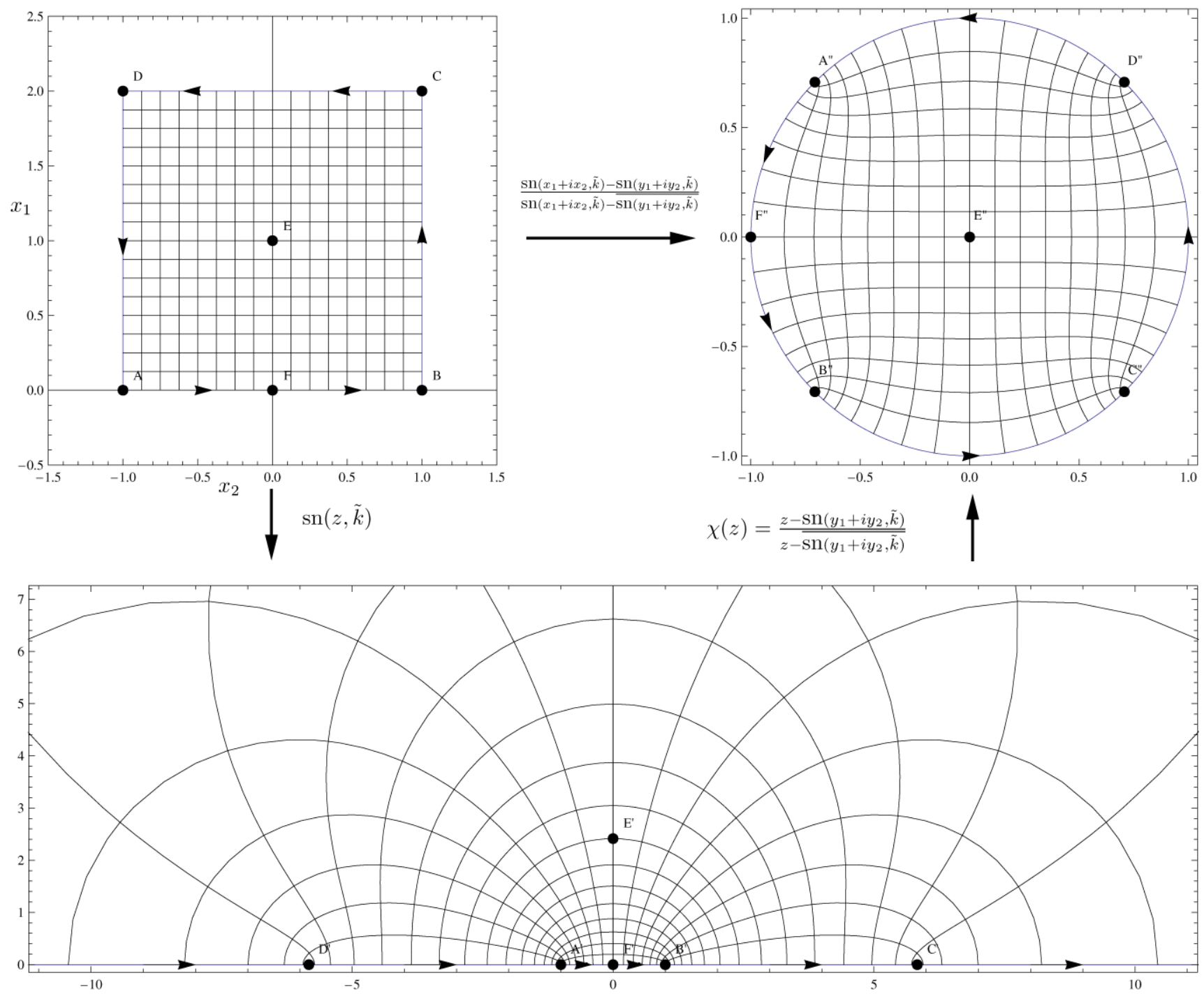

Fig. 9 Mapping from a square (top left) to the upper half space in the complex plane (bottom) followed by a mapping to the disc (uptop right) is used to obtain the Green's function of the Dirichlet operator. In this particular example $a=1, b=2$, and $\left(y_{1}, y_{2}\right)=(0,1)$

where the scalar factor in front of the right Laplacian is the inverse Jacobian:

$$
\begin{aligned}
\left|\mathbf{F}^{\prime}(\mathbf{x})\right|^{-1} & =\left(\operatorname{det} \mathbf{F}^{\prime}(\mathbf{x})\right)^{-1} \\
& =\left(\left(\frac{\partial F_{1}}{\partial x_{1}}(\mathbf{x})\right)^{2}+\left(\frac{\partial F_{2}}{\partial x_{1}}(\mathbf{x})\right)^{2}\right)^{-1} \\
& =\left|F^{\prime}\left(x_{1}+i x_{2}\right)\right|^{-1}
\end{aligned}
$$

for all $\mathbf{x}=\left(x_{1}, x_{2}\right) \in \Omega$.

Now $\tilde{G}(\mathbf{u}, \mathbf{0})=\frac{-1}{2 \pi} \log \|\mathbf{u}\|$ is the unique Green's function with Dirichlet boundary conditions on the disk $\overline{B_{\mathbf{0}, 1}}$ with singularity at $\mathbf{0}$ and our Green's function is given by
$\check{G}=\tilde{G} \circ \mathbf{F}$, i.e.

$$
\begin{aligned}
\check{G} & (\mathbf{x}, \mathbf{y}) \\
& =-\frac{1}{2 \pi} \log \left|(\chi \circ \operatorname{sn}(\cdot, \tilde{k}))\left(x_{1}+i x_{2}\right)\right| \\
& =-\frac{1}{2 \pi} \log \left|\frac{\operatorname{sn}\left(x_{1}+i x_{2}, \tilde{k}\right)-\operatorname{sn}\left(y_{1}+i y_{2}, \tilde{k}\right)}{\operatorname{sn}\left(x_{1}+i x_{2}, \tilde{k}\right)-\overline{\operatorname{sn}\left(y_{1}+i y_{2}, \tilde{k}\right)}}\right| .
\end{aligned}
$$

In (63) and (64) we assumed a certain scaling of $a$ and $b$ such that $C=1$. To obtain the Green's function for the correctly scaled domain we can simply apply an isotropic scaling to the Green's function found in (69). Hence we obtain for the Green's function $G_{a, b}: \Omega \times \Omega \rightarrow \mathbb{R}$ of the Dirichlet operator $\mathcal{D}$, 


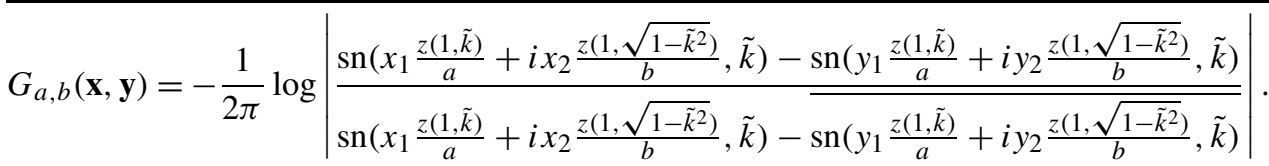

Here we applied the following mappings: $x_{1} \mapsto \frac{x_{1} \operatorname{sn}(1, \tilde{k})}{a}$, $x_{2} \mapsto \frac{x_{2} \operatorname{sn}\left(1, \sqrt{1-\tilde{k}^{2}}\right)}{b}, y_{1} \mapsto \frac{y_{1} \operatorname{sn}(1, \tilde{k})}{a}$, and $y_{2} \mapsto \frac{y_{2} \operatorname{sn}\left(1, \sqrt{1-\tilde{k}^{2}}\right)}{b}$. This is an isotropic scaling because of (62).

\section{The Green's Function on the Unbounded Domain as a Limit of the Dirichlet Kernel}

Next we shall put a relation between the fundamental solution of the Laplace operator on the unbounded domain and the Green's function on the bounded domain with Dirichlet conditions (i.e. the impuls response of the Dirichlet operator) on the square, i.e. $b=2 a$. Here we shall rewrite the solution (70) for $b=2 a$ as follows

$$
\begin{aligned}
G_{a, b=2 a}(\mathbf{x}, \mathbf{y})= & -\frac{1}{2 \pi} \log |\phi(\beta z)-\phi(\beta v)| \\
& +\frac{1}{2 \pi} \log |\phi(\beta z)-\phi(\overline{\beta v})|,
\end{aligned}
$$

where $z=x_{1}+i x_{2}$ and $v=y_{1}+i y_{2}$ and $\phi(\beta z)=$ $\operatorname{sn}\left(\frac{z(1, \tilde{k})}{a}\left(x_{1}+i x_{2}\right), \tilde{k}\right), \beta=\frac{z(1, \tilde{k})}{a}>0$. We recall that $\tilde{k}$ is the unique solution of (62) with $b=2 a$. Furthermore, we note that $\phi(\bar{v})=\overline{\phi(v)}$ for all $v \in \mathbb{C}$.

By taking the limit $a \rightarrow \infty$ and $b=2 a \rightarrow \infty$ in the rectangular case we arrive at the Dirichlet problem on the upper plane $\left\{\left(x_{1}, x_{2}\right) \in \mathbb{R}^{2} \mid x_{2}>0\right\}$. It is well-known, see e.g. (Kreyszig 1993), that the solution of this problem is given by a superposition of two fundamental solutions (the Green's function of the Laplace operator on $\mathbb{R}^{2}$ ). In this case one fundamental solution is centered with plus sign at $\left(y_{1}, y_{2}\right)$ and one fundamental solution is centered at $\left(y_{1},-y_{2}\right)$ with negative sign. This is based on the well-known Schwarz principle and the fact that the difference of these fundamental solutions is zero at the boundary so that the Dirichlet condition is satisfied. In the field of electro-magnetics this result is obvious since the electric potential due to a negative charge at $\left(y_{1}, y_{2}\right)$ cancels out to the electric potential due to a positive charge at $\left(y_{1},-y_{2}\right)$. From this observation we should get

$$
\begin{aligned}
& \lim _{a \rightarrow \infty} G_{a, b=2 a}(\mathbf{x}, \mathbf{y}) \\
& \quad=-\frac{1}{2 \pi} \log |z-v|+\frac{1}{2 \pi} \log |z-\bar{v}|,
\end{aligned}
$$

which we shall verify next.

The mapping $\phi:[-a, a]+i[0, b] \rightarrow \mathbb{C}$ is analytic and by means of the mean-value theorem there exist $\xi_{z, \beta}^{1} \in$
$B_{\beta v, \beta|z-v|}=\{w \in \mathbb{C}|| w-\beta v|<\beta| z-v \mid\}, \xi_{z, \beta}^{2} \in B_{\overline{\beta v}, \beta|z-\bar{v}|}$ Therefore we have

$$
\begin{aligned}
& \phi(z)=\phi(w)+\phi^{\prime}\left(\xi_{z}^{1}\right)(z-w) \quad \text { and } \\
& \phi(z)=\phi(\bar{w})+\phi^{\prime}\left(\xi_{z}^{2}\right)(z-\bar{w})
\end{aligned}
$$

so that we have

$$
\begin{aligned}
& \lim _{a \rightarrow \infty} G_{a, b=2 a}(\mathbf{x}, \mathbf{y}) \\
& \quad=-\frac{1}{2 \pi}\left(\log \left|\frac{\phi^{\prime}\left(\xi_{z}^{1}\right)}{\phi^{\prime}\left(\xi_{z}^{2}\right)}\right|+\log |z-v|-\log |z-\bar{v}|\right),
\end{aligned}
$$

where we recall that $\beta=\left(\frac{z(1, \tilde{k})}{a}\right)$. As $a \rightarrow \infty$, i.e. $\beta \rightarrow 0$, it follows by the continuity of $z \mapsto \phi^{\prime}(z)$ that $\log \left|\frac{\phi^{\prime}\left(\xi_{z}^{1}\right)}{\phi^{\prime}\left(\xi_{z}^{2}\right)}\right| \rightarrow$ $\log 1=0$, from which the result in (72) follows.

\section{References}

Averbuch, A., Israeli, M., \& Vozovoi, L. (1998). A fast Poisson solver of arbitrary order accuracy in rectangular regions. SIAM Journal of Scientific Computing, 19(3), 933-952.

Boersma, J., Jansen, J. K. M., Simons, F. H., \& Sleutel, F. W. (2002). The SIAM 100-dollar, 100-digit challenge-problem 10, SIAM News, January 2002. http://www.win.tue.nl/casa/ meetings/special/siamcontest/problem10.pdf.

Butterworth, S. (1930). On the theory of filter amplifiers. Wireless Engineer, 7, 536-541.

Candes, J. E., Romberg, J., \& Tao, T. (2006). Robust uncertainty principles: Exact signal reconstruction from highly incomplete frequency information. IEEE Transactions on Information Theory, $52(2), 489-509$

Damon, J. (1995). Local Morse theory for solutions to the heat equation and Gaussian blurring. Journal of Differential Equations, 115(2), $368-401$.

Daubechies, I. (1992). CBMS-NSF regional conference series in applied mathematics: Vol. 61. Ten Lectures on Wavelets (8th ed.).

Duchon, J. (1977). Splines minimizing rotation-invariant semi-norms in Sobolev spaces. Berlin: Springer.

Duits, R. (2005). Perceptual organization in image analysis. PhD thesis. Eindhoven University of Technology. http://www.bmi2. bmt.tue.nl/Image-Analysis/People/RDuits/THESISRDUITS.pdf.

Duits, R., Felsberg, M., Florack, L. M. J., \& Platel, B. (2003). $\alpha$ scale spaces on a bounded domain. In L. Griffin, \& M. Lillholm (Eds.), Scale space methods in computer vision, 4th international conference, scale space 2003. Isle of Skye, UK, June 2003 (pp. 494 510). Springer.

Duits, R., Florack, L. M. J., ter Haar Romeny, B. M., \& de Graaf, J. (2004). On the axioms of scale space theory. Journal of Mathematical Imaging and Vision, 20, 267-298.

Florack, L. M. J., \& Kuijper, A. (2000). The topological structure of scale-space images. Journal of Mathematical Imaging and Vision, 12(1), 65-79. 
Georgiev, T. (2005). Relighting, retinex theory, and perceived gradients. In Proceedings of mirage 2005, March 2005.

Gilmore, R. (1993). Catastrophe theory for scientists and engineers. New York: Dover. Originally published by John Wiley \& Sons, New York, 1981.

Gradshteyn, I. S., \& Ryzhik, I. M. (1994). Table of integrals, series, and products (5th edn.). Boston: Academic Press. Edited by A. Jeffrey.

Janssen, B. J., Kanters, F. M. W., Duits, R., Florack, L. M. J., \& ter Haar Romeny, B. M. (2006). A linear image reconstruction framework based on Sobolev type inner products. International Journal of Computer Vision, 70(3), 231-240.

Kanters, F. M. W., Florack, L. M. J., Duits, R., \& Platel, B. (2004). Scalespaceviz: Visualizing $\alpha$-scale spaces. Demonstration software. http://bmia.bmt.tue.nl/people/FKanters/Software/ ScaleSpaceViz.html.

Koenderink, J. J. (1984). The structure of images. Biological Cybernetics, 50, 363-370.

Kreyszig, E. (1993). Advanced engineering mathematics. New York: Wiley.

Kybic, J., Blu, T., \& Unser, M. (2002). Generalized sampling: a variational approach-part I: Theory. IEEE Transactions on Signal Processing, 50, 1965-1976.

Kybic, J., Blu, T., \& Unser, M. (2002). Generalized sampling: a variational approach - part II: Applications. IEEE Transactions on Signal Processing, 50, 1977-1985.

Lillholm, M., Nielsen, M., \& Griffin, L. D. (2003). Feature-based image analysis. International Journal of Computer Vision, 52(2/3), 73-95.
Lorensen, W. E., \& Cline, H. E. (1987). Marching cubes: A high resolution 3d surface construction algorithm. In Computer graphics, proceedings of SIGGRAPH' 87 (Vol. 21, pp. 163-169).

Mallat, S. (1998). A wavelet tour of signal processing. New York: Academic Press.

Nielsen, M., \& Lillholm, M. (2001). What do features tell about images? In Scale-space and morphology in computer vision: Proceedings of the third international conference (pp. 39-50). Springer.

Papoulis, A. (1977). Generalized sampling expansion. IEEE Transactions on Circuits and Systems, 24, 652-654.

Platel, B. (2007). Exploring the deep structure of images. $\mathrm{PhD}$ thesis. Eindhoven University of Technology. http://yp.wtb.tue.nl/ pdfs/8198.pdf.

Press, W. H., Flannery, B. P., Teukolsky, S. A., \& Vetterling, W. T. (1988). Numerical Recipes in C (1st ed.). Cambridge: Cambridge University Press.

Shannon, C. E. Communication in the presence of noise (1949). In Proc. IRE (Vol. 37, pp. 10-21). January 1949.

Unser, M. (2000). Sampling-50 years after shannon. Proceedings of the IEEE, 88(4), 569-587.

Whittaker, E. T., \& Watson, G. N. (1946). Modern analysis (4th ed.). Cambridge: Cambridge University Press.

Yamatani, K., \& Saito, N. (2006). Improvement of DCT-based compression algorithms using Poisson's equation. IEEE Transactions on Image Processing, 15(12), 3672-3689.

Yosida, K. (1980). Functional analysis (6th ed.). Berlin: Springer. 